Please cite this paper as follows:

Chein-Shan Liu and Hong-Ki Hong, Using Comparison Theorem to Compare Corotational Stress Rates in the Model of Perfect Elastoplasticity, International Journal of Solids and Structures, Vol.38, pp.2969-2987, 2001. 


\title{
Using comparison theorem to compare corotational stress rates in the model of perfect elastoplasticity
}

\author{
Chein-Shan Liu ${ }^{\mathrm{a}, *}$, Hong-Ki Hong ${ }^{\mathrm{b}}$ \\ ${ }^{a}$ Department of Mechanical and Marine Engineering, National Taiwan Ocean University, Keelung 202-24, Taiwan, ROC \\ ${ }^{\mathrm{b}}$ Department of Civil Engineering, National Taiwan University, Taipei 106-17, Taiwan, ROC
}

Received 13 June 1999; in revised form 9 May 2000

\begin{abstract}
For the simple shear problem of a perfectly elastoplastic body, we convert the non-linear governing equations into a third order linear differential system, then into a second order linear differential system, and further into a SturmLiouville equation. Thus Sturm's comparison theorem can be employed and extended to compare the simple shear responses based on different objective corotational stress rates. It is proved that the rates of Jaumann, Green-Naghdi, Sowerby-Chu, Xiao-Bruhns-Meyers, and Lee-Mallett-Wertheimer render non-oscillatory stress responses, with the Jaumann equation as a Sturm majorant for the other four equations. For an objective corotational stress rate with the general plane spin a sufficient non-oscillation criterion is found to be that the plane spin must not exceed the shear strain rate. () 2001 Elsevier Science Ltd. All rights reserved.
\end{abstract}

Keywords: Objective corotational stress rates; Perfect elastoplasticity; Lie algebra isomorphism; Sturm comparison theorem; Sturm majorant

\section{Introduction}

The study of objective stress rates is one of the major topics in constitutive modeling. Dienes (1979) was the first to discover that the model of hypoelasticity based on the Jaumann stress rate may result in oscillatory responses to simple shearing. After that, Nagteggal and de Jong (1982) applied the Jaumann rate to the kinematic hardening model and found similar oscillatory behavior. Due to this drawback of the Jaumann rate, considerable efforts have since been made to deal with the problem of choosing an appropriate objective stress rate in rate type constitutive models (see, e.g., Johnson and Bammann, 1984; Atluri, 1984; Metzger and Dubey, 1987; Szabó and Balla, 1989).

\footnotetext{
${ }^{*}$ Corresponding author. Address: Jung He P.O. Box 3-297, Taipei 235, Taiwan, ROC. Tel.: +886-2-2366-1931; fax: +886-2-23622975.

E-mail address: cheinliu@ms47.hinet.net (C.-S. Liu).
} 
Several objective stress rates have been suggested and shown to be plausible by subjecting the constitutive models based on those rates to simple shear deformation. However, a convincing methodology is still lacking for analytically confirming whether a stress rate will render the responses oscillatory or not, and criteria are also lacking for picking out appropriate stress rates. These do pose serious difficulties. Usually the equations encountered are quite complicated, especially for large deformation plasticity models, which are governed by highly non-linear equations; as a consequence, numerical methods were employed to integrate the rate type constitutive equations for different objective stress rates, and the numerical results were then compared between the investigated stress rates (see, e.g., Nagteggal and de Jong, 1982; Moss, 1984; Atluri, 1984; Szabó and Balla, 1989). However, those comparisons gave no answers to questions such as: Why does the Jaumann stress rate used in the model of hypoelasticity render the responses oscillatory, but in the model of perfect elastoplasticity render the responses non-oscillatory? What objective stress rates will render the responses oscillatory, and what else non-oscillatory?

Liu and Hong (2000) recently proposed to use and extend Sturm's comparison theorem to examine the qualitative behavior of hypoelasticity equations. In that paper oscillation and non-oscillation criteria were set up along with the assertions that, among the corotational stress rates examined, the logarithmic stress rate advocated by Xiao et al. (1997a,b) renders its homogeneous equation to be a non-oscillatory majorant, the rate of Jaumann (1911) renders its equation to be an oscillatory minorant, and the rate of Green and Naghdi (1965) renders its homogeneous equation to be a disconjugate majorant. Along this line of thought, in this paper we intend to use Sturm's comparison theorem to study the general influence of the objective corotational stress rates on the simple shear problem of perfect elastoplasticity. Before one can exploit the theorem, the equations of the problem must be subjected to three steps of conversions. They are converted first to a third order linear system in Sections 3 and 6, then to a second order linear system in Sections 9-11, and finally to a Sturm-Liouville equation in Section 11. After the conversions, the main themes of the paper are considered in Sections 7 and 11-13 with Theorems 1 and 2 summarizing the main results.

\section{Constitutive model}

The constitutive law proposed by Prandtl and Reuss can be enlarged to take account of large deformation as in the following (Hong and Liu, 1999):

$$
\begin{aligned}
& \mathbf{D}=\mathbf{D}^{\mathrm{e}}+\mathbf{D}^{\mathrm{p}}, \\
& \dot{\mathbf{s}}=2 G \mathbf{D}^{\mathrm{e}}, \\
& \mathbf{s} \dot{\bar{\gamma}}^{\mathrm{p}}=2 \tau_{y} \mathbf{D}^{\mathrm{p}}, \\
& \|\mathbf{s}\| \leqslant \sqrt{2} \tau_{y}, \\
& \dot{\bar{\gamma}}^{\mathrm{p}} \geqslant 0, \\
& \|\mathbf{s}\| \dot{\bar{\gamma}}^{\mathrm{p}}=\sqrt{2} \tau_{y} \dot{\bar{\gamma}}^{\mathrm{p}}
\end{aligned}
$$

in which the two material constants, namely the shear modulus $G$ and the shear yield stress (or strength) $\tau_{y}$, are determined experimentally and both are assumed to be positive. The boldfaced symbols $\mathbf{D}, \mathbf{D}^{\mathrm{e}}, \mathbf{D}^{\mathrm{p}}$ and $\mathbf{s}$ stand for "the deviatoric " parts of" the deformation rate, elastic deformation rate, plastic deformation rate,

\footnotetext{
${ }^{1}$ Since under simple shear the volumetric parts vanish, "the deviatoric parts of" will be dropped from Section 5 on.
} 
and Cauchy's stress, respectively, all being symmetric and traceless tensors of the second rank, whereas $\bar{\gamma}^{\mathrm{p}}$ is a scalar, called the equivalent shear plastic engineering strain. As usual the Euclidean norm of a tensor, say $\mathbf{s}$, is represented by $\|\mathbf{s}\|:=\sqrt{\mathbf{s} \cdot \mathbf{s}}$, where a dot is placed between two tensors of the same ranks to denote their Euclidean inner product.

A superimposed dot denotes (material) differentiation with respect to time $t$, that is $\mathrm{d} / \mathrm{d} t$, and a surmounted circle "o" on $\mathbf{s}$ represents a Lie derivative of $\mathbf{s}$ with respect to $\omega$,

$$
\stackrel{\circ}{\mathbf{s}}:=\dot{\mathbf{s}}-\omega \mathbf{s}+\mathbf{s} \omega .
$$

Here $\omega$ is a spin tensor to be elaborated later (see Eq. (21)). In the above rate type model (flow model) of perfect elastoplasticity, one crucial point is the choice of the objective stress rate $\stackrel{\mathbf{s}}{\mathbf{s}}$ Although the use of different stress rates makes no fundamental differences in the mathematical structures of the models, fundamental differences in the material behavior modeled may occur.

\section{A linear representation of the model}

A linear representation of the flow model formulated in Section 2 has been obtained by Hong and Liu (1999) as follows:

$$
\dot{\mathbf{X}}=\mathbf{A X}
$$

where

$$
\mathbf{X}=\left[\begin{array}{c}
\mathbf{X}^{s} \\
X^{0}
\end{array}\right]=\left[\begin{array}{c}
X^{1} \\
X^{2} \\
X^{3} \\
X^{4} \\
X^{5} \\
X^{0}
\end{array}\right]:=\frac{X^{0}}{\tau_{y}}\left[\begin{array}{c}
a_{1} s^{11}+a_{2} s^{22} \\
a_{3} s^{11}+a_{4} s^{22} \\
s^{23} \\
s^{13} \\
s^{12} \\
\tau_{y}
\end{array}\right]
$$

is the augmented stress, and

$$
a_{1}:=\sin \left(\varphi+\frac{\pi}{3}\right), \quad a_{2}:=\sin \varphi, \quad a_{3}:=\cos \left(\varphi+\frac{\pi}{3}\right), \quad a_{4}:=\cos \varphi
$$

with $\varphi$ being any real number. The control matrix $\mathbf{A}$ is subjected to the following switch criteria for plastic and elastic phases:

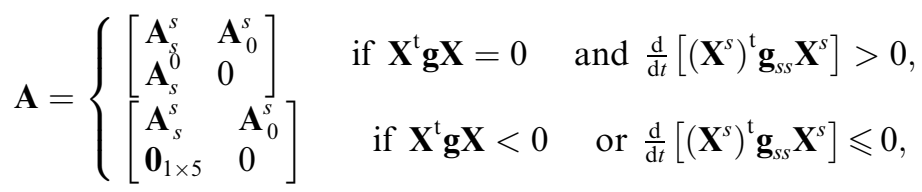

in which 
Table 1

Objective corotational stress rates and spins

\begin{tabular}{ll}
\hline Objective corotational stress rates s̊ & Spin $\boldsymbol{\omega}$ \\
\hline Jaumann (J) & $\mathbf{W}$ \\
Green-Naghdi (GN) & $\mathbf{\Omega}$ \\
Sowerby-Chu (SC) & $\mathbf{\Omega}_{\mathrm{E}}$ \\
Xiao-Bruhns-Meyers (XBM) & $\mathbf{\Omega}^{\log }$ \\
Lee-Mallett-Wertheimer (LMW) & - \\
\hline
\end{tabular}

$$
\begin{aligned}
& \mathbf{A}_{s}^{s}:=\left[\begin{array}{ccccc}
0 & 0 & 2 a_{2} \omega_{23} & 2 a_{1} \omega_{13} & 2\left(a_{1}-a_{2}\right) \omega_{12} \\
& 0 & 2 a_{4} \omega_{23} & 2 a_{3} \omega_{13} & 2\left(a_{3}-a_{4}\right) \omega_{12} \\
& & 0 & -\omega_{12} & -\omega_{13} \\
& \text { skew-sym. } & & 0 & -\omega_{23} \\
& & & & 0
\end{array}\right], \\
& \mathbf{A}_{0}^{s}=\left[\begin{array}{c}
A_{0}^{1} \\
A_{0}^{2} \\
A_{0}^{3} \\
A_{0}^{4} \\
A_{0}^{5}
\end{array}\right]:=\frac{2}{\gamma_{y}}\left[\begin{array}{c}
a_{1} D_{11}+a_{2} D_{22} \\
a_{3} D_{11}+a_{4} D_{22} \\
D_{23} \\
D_{13} \\
D_{12}
\end{array}\right], \\
& \mathbf{A}_{s}^{0}=\left(\mathbf{A}_{0}^{s}\right)^{\mathrm{t}},
\end{aligned}
$$

where $\gamma_{y}:=\tau_{y} / G$ is the shear yield engineering strain, $\omega_{i j}, i, j=1,2,3$, are the components of $\omega$,

$$
\mathbf{g}=\left[\begin{array}{ll}
\mathbf{g}_{s s} & \mathbf{g}_{s 0} \\
\mathbf{g}_{0 s} & \mathrm{~g}_{00}
\end{array}\right]=\left[\begin{array}{cc}
\mathbf{I}_{5} & \mathbf{0}_{5 \times 1} \\
\mathbf{0}_{1 \times 5} & -1
\end{array}\right]
$$

is the Minkowski metric, $\mathbf{I}_{n}$ is the identity matrix of order $n, \mathbf{0}_{m \times n}$ is the $m \times n$ zero matrix, and the superscript $\mathrm{t}$ denotes the transpose.

Note that Eq. (8) is a linear $(5+1)$-dimensional representation of the flow model (1)-(7). The control matrix A organizes the input information of "the deviatoric ${ }^{2}$ part of" the deformation rate tensor D (normalized with respect to the shear yield strain $\gamma_{y} / 2$ ) and the spin tensor $\boldsymbol{\omega}$. Because of the large number $2 / \gamma_{y}$ in $\mathbf{A}_{0}^{s}$, the values of the components of $\mathbf{A}_{s}^{s}$ are much less than those of $\mathbf{A}_{0}^{s}$, and so the major contribution to $\mathbf{X}$ and hence to $\mathbf{s}$ is made by $\mathbf{D}$, not by $\boldsymbol{\omega}$.

\section{Objective corotational stress rates}

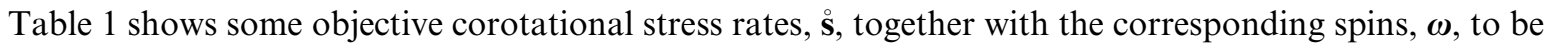
investigated in this paper. First the spins are put in Eq. (7) to obtain the rates and then the rates are put in the constitutive equations (1)-(6) for further study.

The notation used here for the spins $\mathbf{W}, \boldsymbol{\Omega}, \boldsymbol{\Omega}_{\mathrm{E}}$, and $\mathbf{\Omega}^{\log }$ is explained as follows: $\mathbf{W}$ is the skew-symmetric part of the velocity gradient tensor $\mathbf{L}:=\dot{\mathbf{F}}^{-1}$, where $\mathbf{F}$ is the two-point tensor of deformation gradient. $\mathbf{\Omega}:=\dot{\mathbf{R}} \mathbf{R}^{\mathrm{t}}$ is the rate of rotation, where $\mathbf{R}$ is the rotation, the orthogonal tensor in the polar decomposition

\footnotetext{
2 "The deviatoric part of" will be dropped from Section 5 on.
} 
$\mathbf{F}=\mathbf{R} \mathbf{U}=\mathbf{V R}$ of $\mathbf{F} . \boldsymbol{\Omega}_{\mathrm{E}}:=\dot{\mathbf{R}}_{\mathrm{E}} \mathbf{R}_{\mathrm{E}}^{\mathrm{t}}$ is known as the Eulerian spin tensor, where $\mathbf{R}_{\mathrm{E}}$ is the diagonal transformation of $\mathbf{V}$, that is

$$
\mathbf{V}=\mathbf{R}_{\mathrm{E}} \lambda \mathbf{R}_{\mathrm{E}}^{\mathrm{t}}
$$

with $\lambda=\operatorname{diag}\left[\lambda_{1}, \lambda_{2}, \lambda_{3}\right]$ the diagonal tensor containing the eigenvalues, $\lambda_{1}, \lambda_{2}, \lambda_{3}$, of $\mathbf{V}$. The logarithmic spin $\boldsymbol{\Omega}^{\log }$ was introduced recently in order that the deformation rate equals $(\ln \mathbf{V})^{\circ}-\boldsymbol{\Omega}^{\log } \ln \mathbf{V}+(\ln \mathbf{V}) \boldsymbol{\Omega}^{\log }$, where $(\ln \mathbf{V})$ denotes the material time derivative of the Eulerian logarithmic strain tensor $\ln \mathbf{V}$ (see Lehmann et al., 1991; Reinhardt and Dubey, 1996; Xiao et al., 1997a,b). With the logarithmic spin $\mathbf{\Omega}^{\log }$, the logarithmic rate of any Eulerian symmetric tensor, say $\mathbf{S}$, is defined by

$$
\dot{\mathbf{S}}^{\log }:=\dot{\mathbf{S}}-\mathbf{\Omega}^{\log } \mathbf{S}+\mathbf{S} \boldsymbol{\Omega}^{\log }
$$

which indeed generalizes the concept of the objective stress rates in Eq. (7), but specializes $\omega$ to be $\boldsymbol{\Omega}^{\log }$. In particular, if setting $\mathbf{S}$ to be Cauchy's stress ${ }^{3} \boldsymbol{\sigma}$, then one has the logarithmic rate of Cauchy's stress, $\dot{\sigma}^{\log }$, which will be referred to later as the Xiao-Bruhns-Meyers rate. Note that $\boldsymbol{\sigma}^{\circ}=\boldsymbol{\sigma}^{\log }$ if $\boldsymbol{\omega}=\boldsymbol{\Omega}^{\log }$. It is remarkable that the deformation rate can thus be seen to be equal to $(\ln \mathbf{V})^{\log }$.

\section{Simple shear deformation}

In what follows we are going to study a solid body, which is made of material described by the model of perfect elastoplasticity formulated in Section 2, under simple shear deformation, of which the deformation gradient is

$$
\mathbf{F}=\left[\begin{array}{lll}
1 & \gamma & 0 \\
0 & 1 & 0 \\
0 & 0 & 1
\end{array}\right], \quad \gamma \in[0, \infty),
$$

where $\gamma$ is the shear engineering strain. Let

$$
\theta:=\arctan (\gamma / 2), \quad \dot{\theta}=\frac{2 \dot{\gamma}}{\gamma^{2}+4}, \quad \theta \in[0, \pi / 2) .
$$

The related kinematic quantities are listed below:

$$
\begin{aligned}
\mathbf{D} & =\frac{\dot{\gamma}}{2}\left[\begin{array}{lll}
0 & 1 & 0 \\
1 & 0 & 0 \\
0 & 0 & 0
\end{array}\right], \quad \mathbf{W}=\frac{\dot{\gamma}}{2}\left[\begin{array}{ccc}
0 & 1 & 0 \\
-1 & 0 & 0 \\
0 & 0 & 0
\end{array}\right], \quad \boldsymbol{\Omega}=\left[\begin{array}{ccc}
0 & \dot{\theta} & 0 \\
-\dot{\theta} & 0 & 0 \\
0 & 0 & 0
\end{array}\right], \\
\boldsymbol{\Omega}_{\mathrm{E}}=\left[\begin{array}{ccc}
0 & \frac{\dot{\theta}}{2} & 0 \\
-\frac{\dot{\theta}}{2} & 0 & 0 \\
0 & 0 & 0
\end{array}\right], & \boldsymbol{\Omega}^{\log }=\frac{\dot{\gamma}}{2}\left[\begin{array}{ccc}
0 & f(\theta) & 0 \\
-f(\theta) & 0 & 0 \\
0 & 0 & 0
\end{array}\right],
\end{aligned}
$$

where

$$
f(\theta)=\frac{1}{2 \ln ((1+\sin \theta) / \cos \theta)}\left(\sin \theta+\cos ^{2} \theta \ln \frac{1+\sin \theta}{\cos \theta}\right)
$$

as has been derived by Liu and Hong (2000).

\footnotetext{
${ }^{3}$ From Section 5 on, $\boldsymbol{\sigma}=\mathbf{s}$.
} 
The forms of $\mathbf{W}, \boldsymbol{\Omega}, \boldsymbol{\Omega}_{\mathrm{E}}$, and $\boldsymbol{\Omega}^{\log }$ lead us to consider the following general plane spin:

$$
\omega=\frac{\dot{\gamma}}{2}\left[\begin{array}{ccc}
0 & f & 0 \\
-f & 0 & 0 \\
0 & 0 & 0
\end{array}\right]
$$

which includes at least the following as special cases:

1. The Jaumann rate (1911): $f=1$ for $\mathbf{W}$.

2. The Green-Naghdi rate (1965): $f(\gamma)=4 /\left(\gamma^{2}+4\right)$ for $\boldsymbol{\Omega}$.

3. The Sowerby-Chu rate (Sowerby and Chu, 1984): $f(\gamma)=2 /\left(\gamma^{2}+4\right)$ for $\boldsymbol{\Omega}_{\mathrm{E}}$.

4. The Xiao-Bruhns-Meyers rate (1997a,b): $f(\theta)$ as in Eq. (20) for $\mathbf{\Omega}^{\log }$.

5. The Lee-Mallett-Wertheimer rate (Lee et al., 1983): $f(\gamma)=2 /\left(\gamma^{2}+1\right)$. (see also Yang et al., 1992).

From Eq. (21), the plane spin $\omega_{12}=f \dot{\gamma} / 2$. Since $\gamma$ is the shear engineering strain, $\gamma / 2$ is the shear strain and $\dot{\gamma} / 2$ is the shear strain rate, so that $f$ is the ratio of the plane spin $\omega_{12}$ to the shear strain rate $\dot{\gamma} / 2$. For the Jaumann stress rate the plane spin equals the shear strain rate, but for the other four stress rates they are not equal.

Formula (20) is equivalent to ${ }^{4}$

$$
\frac{g(\omega)}{2}=\frac{1}{2\left(1+\omega^{2}\right)}+\frac{\omega}{2 \sqrt{1+\omega^{2}} \operatorname{sh}^{-1} \omega}
$$

Eq. (157) of the article of Bruhns et al. (1999), upon making the change of variables $\omega=\gamma / 2=\tan \theta$ and $\operatorname{sh}^{-1} \omega=\ln \left(\omega+\sqrt{1+\omega^{2}}\right)=\ln ((1+\sin \theta) / \cos \theta)$.

\section{Simple shear equations}

Under simple shear we have

$$
\mathbf{s}=\left[\begin{array}{ccc}
s_{11} & s_{12} & 0 \\
s_{12} & -s_{11} & 0 \\
0 & 0 & 0
\end{array}\right]
$$

Thus Eq. (8) reduces to

$$
\frac{\mathrm{d}}{\mathrm{d} t}\left[\begin{array}{l}
X^{1} \\
X^{5} \\
X^{0}
\end{array}\right]=\left[\begin{array}{ccc}
0 & 2 \omega_{12} & 0 \\
-2 \omega_{12} & 0 & \frac{2}{\gamma_{y}} D_{12} \\
0 & \frac{2}{\gamma_{y}} D_{12} & 0
\end{array}\right]\left[\begin{array}{l}
X^{1} \\
X^{5} \\
X^{0}
\end{array}\right],
$$

where we have chosen $\varphi=11 \pi / 6$ and hence $a_{1}-a_{2}=1$ in Eq. (10). Substituting Eq. (19) for D and Eq. (21) for $\omega$ in Eq. (23), we have

$$
\frac{\mathrm{d}}{\mathrm{d} \gamma}\left[\begin{array}{l}
X^{1} \\
X^{5} \\
X^{0}
\end{array}\right]=\left[\begin{array}{ccc}
0 & f & 0 \\
-f & 0 & \frac{1}{\gamma_{y}} \\
0 & \frac{1}{\gamma_{y}} & 0
\end{array}\right]\left[\begin{array}{c}
X^{1} \\
X^{5} \\
X^{0}
\end{array}\right]
$$

\footnotetext{
${ }^{4}$ The authors would like to thank one reviewer who drew the authors' attention to this equivalence. For notation, the $\omega$ is neither $\omega$ nor $\omega_{i j}$ at all, but is $\gamma / 2$.
} 


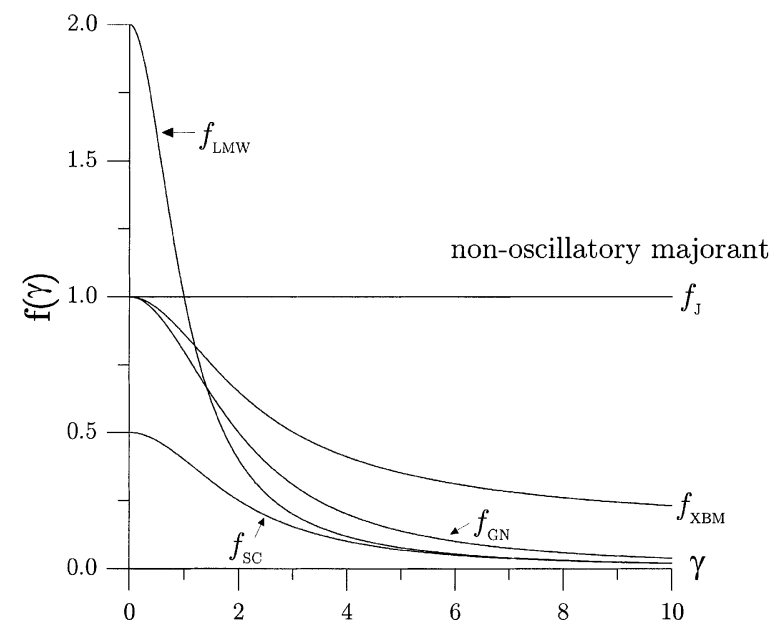

Fig. 1. The five $f(\gamma)$ 's of Jaumann, Green-Naghdi, Sowerby-Chu, Xiao-Bruhns-Meyers, and Lee-Mallett-Wertheimer.

Hence the axial and shear stress components,

$$
s^{11}=\frac{\tau_{y} X^{1}}{X^{0}}, \quad s^{12}=\frac{\tau_{y} X^{5}}{X^{0}},
$$

are governed by the following differential equations:

$$
\begin{aligned}
\frac{\mathrm{d}}{\mathrm{d} \gamma} s^{11} & =f(\gamma) s^{12}-\frac{1}{\gamma_{y} \tau_{y}} s^{11} s^{12}, \\
\frac{\mathrm{d}}{\mathrm{d} \gamma} s^{12} & =-f(\gamma) s^{11}-\frac{1}{\gamma_{y} \tau_{y}}\left(s^{12}\right)^{2}+\frac{\tau_{y}}{\gamma_{y}} .
\end{aligned}
$$

Let the right-hand sides of Eqs. (26) and (27) equal zeros; we obtain the limiting values of the responses

$$
\lim _{\gamma \rightarrow \infty} s^{11}=\gamma_{y} \tau_{y} f(\infty), \quad \lim _{\gamma \rightarrow \infty} s^{12}=\sqrt{\tau_{y}^{2}-\gamma_{y}^{2} \tau_{y}^{2} f^{2}(\infty)} .
$$

The asymptotic behavior of the stresses is seen to be controlled by $f(\infty)$. The five $f$ 's listed in the previous section are compared in Fig. 1, which shows that most of $f$ 's (except that of Jaumann) tend to zero as $\gamma \rightarrow \infty$.

\section{Closed-form solutions of the Jaumann equation}

Differentiating the second equation of Eq. (24) and using the other two equations thereof, we obtain

$$
\frac{\mathrm{d}^{2} X^{5}}{\mathrm{~d} \gamma^{2}}=\frac{f^{\prime}}{f} \frac{\mathrm{d} X^{5}}{\mathrm{~d} \gamma}-\frac{f^{\prime}}{\gamma_{y} f} X^{0}+\left(\frac{1}{\gamma_{y}^{2}}-f^{2}\right) X^{5}
$$

in which the primes stand for differentiation with respect to $\gamma$. For the Jaumann stress rate, $f=1$ and $f^{\prime}=0$, so Eq. (29) reducing to 


$$
\frac{\mathrm{d}^{2} X^{5}}{\mathrm{~d} \gamma^{2}}+\left(1-\frac{1}{\gamma_{y}^{2}}\right) X^{5}=0 .
$$

This equation indicates that, unless $\gamma_{y}>1, X^{5}$ and hence $s^{12}$ will not oscillate. However, for most materials, the theoretical upper limit of $\gamma_{y}$ is $1 / 15$ (see e.g., Hirth and Lothe, 1982); therefore, $\gamma_{y}<1$. From Eqs. (30), (24) and (25) the responses can be derived as follows:

$$
\begin{aligned}
& \frac{s^{11}}{\tau_{y}}=\frac{m \gamma_{y} c_{4}+\gamma_{y} c_{1} \exp \left[m\left(\gamma-\gamma_{\text {on }}\right)\right]-\gamma_{y} c_{2} \exp \left[-m\left(\gamma-\gamma_{\text {on }}\right)\right]}{m \gamma_{y} c_{3}+c_{1} \exp \left[m\left(\gamma-\gamma_{\text {on }}\right)\right]-c_{2} \exp \left[-m\left(\gamma-\gamma_{\text {on }}\right)\right]}, \\
& \frac{s^{12}}{\tau_{y}}=\frac{m \gamma_{y} c_{1} \exp \left[m\left(\gamma-\gamma_{\text {on }}\right)\right]+m \gamma_{y} c_{2} \exp \left[-m\left(\gamma-\gamma_{\text {on }}\right)\right]}{m \gamma_{y} c_{3}+c_{1} \exp \left[m\left(\gamma-\gamma_{\text {on }}\right)\right]-c_{2} \exp \left[-m\left(\gamma-\gamma_{\text {on }}\right)\right]},
\end{aligned}
$$

where

$$
\begin{aligned}
& m=\sqrt{\frac{1}{\gamma_{y}^{2}}-1}, \quad c_{1}=\frac{1}{2 m \gamma_{y}}+\frac{s^{12}\left(\gamma_{\mathrm{on}}\right)}{2 \tau_{y}}-\frac{s^{11}\left(\gamma_{\mathrm{on}}\right)}{2 m \tau_{y}}, \quad c_{2}=-\frac{1}{2 m \gamma_{y}}+\frac{s^{12}\left(\gamma_{\mathrm{on}}\right)}{2 \tau_{y}}+\frac{s^{11}\left(\gamma_{\mathrm{on}}\right)}{2 m \tau_{y}}, \\
& c_{3}=1-\frac{1}{m \gamma_{y}}\left[\frac{1}{m \gamma_{y}}-\frac{s^{11}\left(\gamma_{\mathrm{on}}\right)}{m \tau_{y}}\right], \quad c_{4}=\frac{c_{3}}{\gamma_{y}}
\end{aligned}
$$

in which $\gamma=\gamma(t)$ and $\gamma_{\text {on }}=\gamma\left(t_{\text {on }}\right)$, where $t$ and $t_{\text {on }}$ are the current time and the switch-on time of plastic deformation. For $\gamma_{\text {on }} \leqslant \gamma<\infty$ the body is in the plastic phase, while for $0 \leqslant \gamma<\gamma_{\text {on }}$ it is in the elastic phase. Note that $\gamma_{\text {on }} \neq \gamma_{y}$ unless the initial stresses are zeros and Eq. (2) is replaced by $\dot{\mathbf{s}}=2 G \mathbf{D}^{\mathbf{e}}$. Formulae (31) and (32) (and also Fig. 2) confirm that the axial and shear responses are non-oscillatory. ${ }^{5}$

Note that Eq. (24) is for perfect elastoplasticity; on the other hand, for hypoelasticity, it becomes

$$
\frac{\mathrm{d}}{\mathrm{d} \gamma}\left[\begin{array}{l}
X^{1} \\
X^{5} \\
X^{0}
\end{array}\right]=\left[\begin{array}{ccc}
0 & f & 0 \\
-f & 0 & \frac{1}{\gamma_{y}} \\
0 & 0 & 0
\end{array}\right]\left[\begin{array}{c}
X^{1} \\
X^{5} \\
X^{0}
\end{array}\right]
$$

which, for the Jaumann stress rate, leads to $X^{0}=1$ and

$$
\frac{\mathrm{d}^{2} X^{5}}{\mathrm{~d} \gamma^{2}}+X^{5}=0
$$

Obviously, this equation indicates that $X^{5}$ and hence $s^{12}$ for hypoelasticity are oscillatory. ${ }^{6}$ The contrast between Eqs. (34) and (30) (and between Eqs. (33) and (24)) reveals that the fundamental difference of the mathematical structures between hypoelasticity and elastoplasticity and the physical limit $0<\gamma_{y}<1$ of the plastic yield are the major factors which render the responses qualitatively different for the Jaumann rate.

\section{Consistency scheme}

From Eq. (25) it follows that

$$
\left(s^{11}\right)^{2}+\left(s^{12}\right)^{2}=\tau_{y}^{2} \Longleftrightarrow\left(X^{1}\right)^{2}+\left(X^{5}\right)^{2}-\left(X^{0}\right)^{2}=0
$$

\footnotetext{
${ }^{5}$ The precise meaning (definition) of non-oscillatory will be given in Section 11 .

${ }^{6}$ The precise meaning (definition) of oscillatory will be given in Section 11.
} 

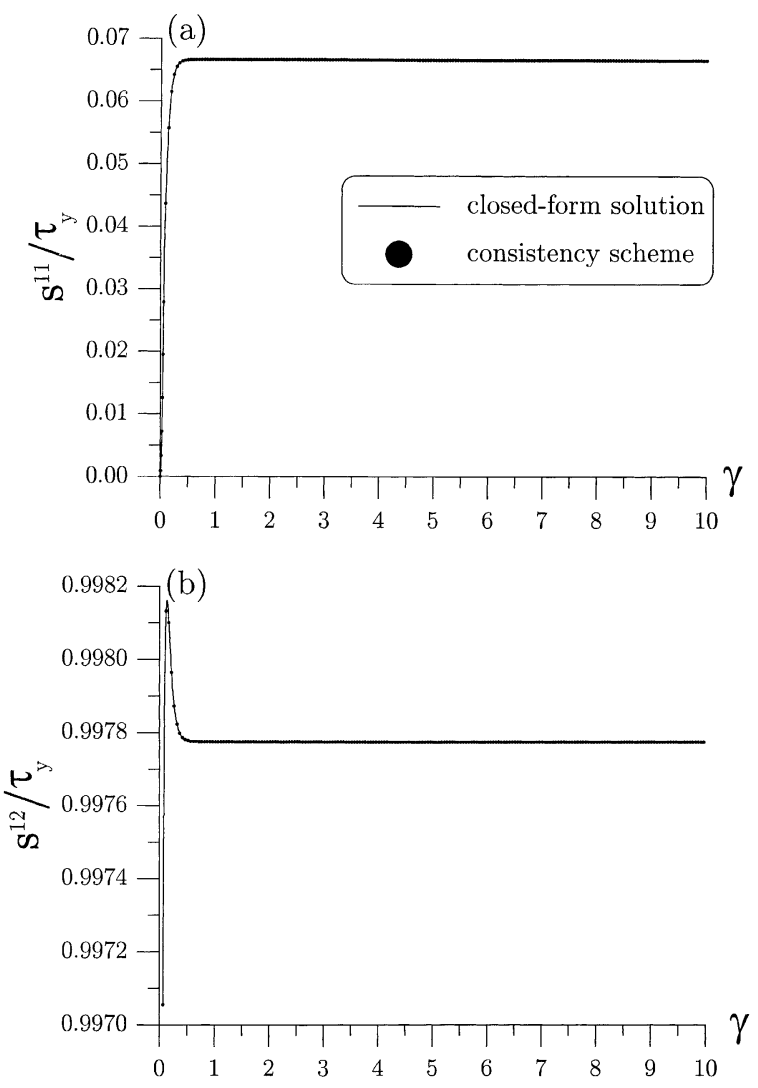

Fig. 2. Axial and shear stress responses for the simple shear problem based on the Jaumann rate. Those calculated by the group preserving scheme agree very well with the closed-form solutions.

and so the yield condition in the two-dimensional stress space $\left(s^{11}, s^{12}\right)$ is converted to a null cone condition in the three-dimensional Minkowski space $\left(X^{1}, X^{5}, X^{0}\right) \in \mathbb{M}^{3}$, which endows the indefinite metric

$$
\mathbf{g}=\left[\begin{array}{cc}
\mathbf{I}_{2} & \mathbf{0}_{2 \times 1} \\
\mathbf{0}_{1 \times 2} & -1
\end{array}\right] .
$$

The control matrix

$$
\mathbf{A}=\dot{\gamma}\left[\begin{array}{ccc}
0 & f & 0 \\
-f & 0 & \frac{1}{\gamma_{y}} \\
0 & \frac{1}{\gamma_{y}} & 0
\end{array}\right]
$$

in Eq. (24) satisfies

$$
\mathbf{A}^{\mathrm{t}} \mathbf{g}+\mathbf{g A}=\mathbf{0},
$$

and hence is an element of the Lie algebra so $(2,1)$. Correspondingly, the fundamental solution $\mathbf{G}$ of Eq. (24) satisfies (see e.g., Hong and Liu, 1999, 2000) 

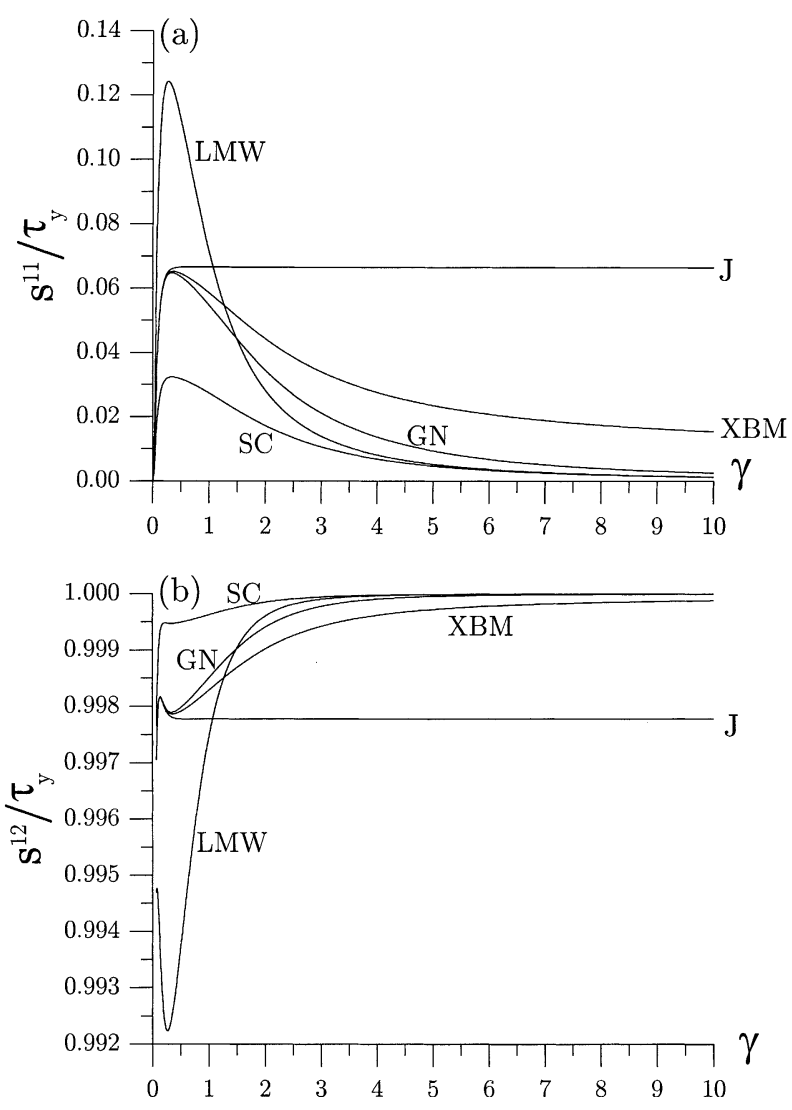

Fig. 3. Axial and shear stress responses for the simple shear problem based on the rates of Jaumann, Green-Naghdi, Sowerby-Chu, Xiao-Bruhns-Meyers, and Lee-Mallett-Wertheimer.

$$
\begin{aligned}
& \mathbf{G}^{\mathbf{t}} \mathbf{g G}=\mathbf{g}, \\
& \operatorname{det} \mathbf{G}=1, \\
& G_{0}^{0} \geqslant 1
\end{aligned}
$$

and hence is an element of the proper orthochronous Lorentz group $\mathrm{SO}_{\mathrm{o}}(2,1)$. This leads us to use the following group preserving scheme for Eq. (24):

$$
\mathbf{X}_{n+1}=\left(\mathbf{I}_{3}-\tau \mathbf{A}\right)^{-1}\left(\mathbf{I}_{3}+\tau \mathbf{A}\right) \mathbf{X}_{n}=\left[\mathbf{I}_{3}+2 \tau\left(\mathbf{I}_{3}-\tau \mathbf{A}\right)^{-1} \mathbf{A}\right] \mathbf{X}_{n},
$$

where $\mathbf{X}_{n}$ denotes $\mathbf{X}\left(\gamma_{n}\right)$ and $\tau:=\Delta \gamma / 2=\left(\gamma_{n+1}-\gamma_{n}\right) / 2$. It is easy to check that this transform preserves the properties (39)-(41). This scheme may be specifically called a consistency scheme, since it is capable, among other benefits derivable from the group properties, of updating the stress point to be automatically located on the yield surface at the end of each step in the plastic phase without any iterative calculations; that is, the consistency condition is fulfilled automatically and exactly.

In Fig. 2 the closed-form solutions of the Jaumann equation are plotted to check the numerical solutions using the above scheme. It can be seen that this scheme is very accurate. Fig. 3 shows the five $s^{11}$ 's and $s^{12}$ 's for the equations of Jaumann, Green-Naghdi, Sowerby-Chu, Xiao-Bruhns-Meyers and Lee-MallettWertheimer. Before further comparing the five corotational stress rates, let us manage to reduce the order of system (24) in the following two sections. 
9. Spinor map from $\operatorname{SL}(2, \mathbb{R})$ onto $\mathrm{SO}_{0}(2,1)$

Any $2 \times 2$ real symmetric matrix $\mathbf{H}$ can be written as

$$
\mathbf{H}=\left[\begin{array}{cc}
x^{0}+x^{1} & x^{2} \\
x^{2} & x^{0}-x^{1}
\end{array}\right]
$$

where $x^{0}, x^{1}$ and $x^{2}$ are real numbers. ${ }^{7}$ The determinant of $\mathbf{H}$ is $\left(x^{0}\right)^{2}-\left(x^{1}\right)^{2}-\left(x^{2}\right)^{2}$.

The group $\operatorname{SL}(2, \mathbb{R})$ consists of all real $2 \times 2$ matrices $\mathbf{U}$ with unit determinant

$$
\mathbf{U}=\left[\begin{array}{ll}
U_{1}^{1} & U_{2}^{1} \\
U_{1}^{2} & U_{2}^{2}
\end{array}\right], \quad \operatorname{det} \mathbf{U}=1
$$

and is a subgroup of $\operatorname{SL}(2, \mathbb{C})$, elements of which are often called spin transformations, so that $\mathbf{U}$ is a spin transformation (matrix). Since $\mathbf{H}$ is symmetrical, it is obvious that $\mathbf{U} \mathbf{H} \mathbf{U}^{\mathrm{t}}$ is also a $2 \times 2$ real symmetrical matrix. This leads us to write

$$
\hat{\mathbf{H}}=\mathbf{U H U}^{\mathrm{t}} \text {, }
$$

namely,

$$
\left[\begin{array}{cc}
\hat{x}^{0}+\hat{x}^{1} & \hat{x}^{2} \\
\hat{x}^{2} & \hat{x}^{0}-\hat{x}^{1}
\end{array}\right]=\left[\begin{array}{cc}
U_{1}^{1} & U_{2}^{1} \\
U_{1}^{2} & U_{2}^{2}
\end{array}\right]\left[\begin{array}{cc}
x^{0}+x^{1} & x^{2} \\
x^{2} & x^{0}-x^{1}
\end{array}\right]\left[\begin{array}{cc}
U_{1}^{1} & U_{1}^{2} \\
U_{2}^{1} & U_{2}^{2}
\end{array}\right] .
$$

Taking the determinants of both sides and using $\operatorname{det} \mathbf{U}=\operatorname{det} \mathbf{U}^{\mathrm{t}}=1$, one readily obtains $\left(\hat{x}^{1}\right)^{2}+$ $\left(\hat{x}^{2}\right)^{2}-\left(\hat{x}^{0}\right)^{2}=\left(x^{1}\right)^{2}+\left(x^{2}\right)^{2}-\left(x^{0}\right)^{2}$, ensuring that the length (more precisely, the Minkowski separation) of the $(2+1)$-vector $\mathbf{x}=\left(x^{1}, x^{2}, x^{0}\right)^{\mathrm{t}}$ is preserved by the spin transformation $\mathbf{U}: \mathbf{H} \mapsto \hat{\mathbf{H}}$. Indeed, the spin transformation $\mathbf{U}: \mathbf{H} \mapsto \hat{\mathbf{H}}$ induces a proper orthochronous Lorentz transformation $\mathbf{G}: \mathbf{x} \mapsto \hat{\mathbf{x}}$, which is an element of $\mathrm{SO}_{\mathrm{o}}(2,1)$. Eq. (43) may be rearranged as

$$
\left[\begin{array}{l}
H_{11} \\
H_{12} \\
H_{22}
\end{array}\right]=\mathbf{C}\left[\begin{array}{l}
x^{1} \\
x^{2} \\
x^{0}
\end{array}\right]
$$

where

$$
\mathbf{C}:=\left[\begin{array}{ccc}
1 & 0 & 1 \\
0 & 1 & 0 \\
-1 & 0 & 1
\end{array}\right]
$$

Then Eq. (45) is equivalent to

$$
\left[\begin{array}{l}
\hat{H}_{11} \\
\hat{H}_{12} \\
\hat{H}_{22}
\end{array}\right]=\mathbf{R}\left[\begin{array}{l}
H_{11} \\
H_{12} \\
H_{22}
\end{array}\right]
$$

where

$$
\mathbf{R}:=\left[\begin{array}{ccc}
\left(U_{1}^{1}\right)^{2} & 2 U_{1}^{1} U_{2}^{1} & \left(U_{2}^{1}\right)^{2} \\
U_{1}^{1} U_{1}^{2} & U_{2}^{1} U_{1}^{2}+U_{1}^{1} U_{2}^{2} & U_{2}^{1} U_{2}^{2} \\
\left(U_{1}^{2}\right)^{2} & 2 U_{1}^{2} U_{2}^{2} & \left(U_{2}^{2}\right)^{2}
\end{array}\right]
$$

\footnotetext{
${ }^{7}$ For notation, do not mix up the lower case symbols $x^{0}, x^{1}, x^{2}$ with the capital symbols $X^{0}, X^{1}, X^{2}$.
} 
Applying

$$
\mathbf{C}^{-1}=\frac{1}{2}\left[\begin{array}{ccc}
1 & 0 & -1 \\
0 & 2 & 0 \\
1 & 0 & 1
\end{array}\right]
$$

to both sides of Eq. (48) and noting Eq. (46) we have

$$
\left[\begin{array}{c}
\hat{x}^{1} \\
\hat{x}^{2} \\
\hat{x}^{0}
\end{array}\right]=\mathbf{C}^{-1} \mathbf{R C}\left[\begin{array}{l}
x^{1} \\
x^{2} \\
x^{0}
\end{array}\right] .
$$

Comparing this with the proper orthochronous Lorentz transformation

$$
\left[\begin{array}{c}
\hat{x}^{1} \\
\hat{x}^{2} \\
\hat{x}^{0}
\end{array}\right]=\mathbf{G}\left[\begin{array}{c}
x^{1} \\
x^{2} \\
x^{0}
\end{array}\right]
$$

we have

$$
\mathbf{G}=\mathbf{C}^{-1} \mathbf{R C} \text {. }
$$

Substituting Eq. (50) for $\mathbf{C}^{-1}$, Eq. (49) for $\mathbf{R}$, and Eq. (47) for $\mathbf{C}$ in the above equation, we obtain the spinor map

$$
\begin{aligned}
& G_{1}^{1}=\frac{\left(U_{1}^{1}\right)^{2}-\left(U_{1}^{2}\right)^{2}-\left(U_{2}^{1}\right)^{2}+\left(U_{2}^{2}\right)^{2}}{2}, \\
& G_{2}^{1}=U_{1}^{1} U_{2}^{1}-U_{1}^{2} U_{2}^{2}, \\
& G_{0}^{1}=\frac{\left(U_{1}^{1}\right)^{2}-\left(U_{1}^{2}\right)^{2}+\left(U_{2}^{1}\right)^{2}-\left(U_{2}^{2}\right)^{2}}{2}, \\
& G_{1}^{2}=U_{1}^{1} U_{1}^{2}-U_{2}^{1} U_{2}^{2}, \\
& G_{2}^{2}=U_{1}^{1} U_{2}^{2}+U_{2}^{1} U_{1}^{2}, \\
& G_{0}^{2}=U_{1}^{1} U_{1}^{2}+U_{2}^{1} U_{2}^{2}, \\
& G_{1}^{0}=\frac{\left(U_{1}^{1}\right)^{2}+\left(U_{1}^{2}\right)^{2}-\left(U_{2}^{1}\right)^{2}-\left(U_{2}^{2}\right)^{2}}{2}, \\
& G_{2}^{0}=U_{1}^{1} U_{2}^{1}+U_{1}^{2} U_{2}^{2}, \\
& G_{0}^{0}=\frac{\left(U_{1}^{1}\right)^{2}+\left(U_{1}^{2}\right)^{2}+\left(U_{2}^{1}\right)^{2}+\left(U_{2}^{2}\right)^{2}}{2} .
\end{aligned}
$$

This map is from the group $\operatorname{SL}(2, \mathbb{R})$ onto the group $\mathrm{SO}_{\mathrm{o}}(2,1)$ and is a two-valued representation in the sense that the two spin transformations $\pm \mathbf{U}$ map to the same proper orthochronous Lorentz transformation G. Fig. 4 summarizes the procedure for obtaining the spinor map from $\operatorname{SL}(2, \mathbb{R})$ onto $\operatorname{SO}_{\mathrm{o}}(2,1)$.

With formulae (54)-(62) the $2 \times 2$ matrix $\mathbf{U}$ suffices to determine the $3 \times 3$ matrix $\mathbf{G}$. Therefore, instead of solving the third order fundamental solution equation

$$
\dot{\mathbf{G}}=\mathbf{A G}, \quad \mathbf{G}(0)=\mathbf{I}_{3},
$$

we may solve the second order fundamental solution equation

$$
\dot{\mathbf{U}}=\mathbf{B U}, \quad \mathbf{U}(0)=\mathbf{I}_{2} .
$$




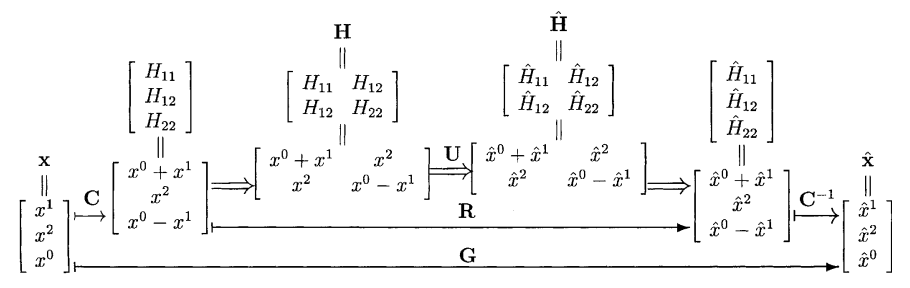

Fig. 4. Algebraic procedure for constructing the spinor map from $\operatorname{SL}(2, \mathbb{R})$ onto $\operatorname{SO}_{\mathrm{o}}(2,1)$.

But we still lack a conversion formula from $\mathbf{B} \in \operatorname{sl}(2, \mathbb{R})$ to $\mathbf{A} \in \operatorname{so}(2,1)$. This will be formulated in the Section 10.

\section{Lie algebra isomorphism of $\operatorname{sl}(2, \mathbb{R})$ onto $\operatorname{so}(2,1)$}

This section relates $\mathbf{A}$ of the third order system (63) to $\mathbf{B}$ of the second order system (64), and vice versa. The relation is indeed a Lie algebra isomorphism of $\operatorname{sl}(2, \mathbb{R})$ onto $\operatorname{so}(2,1)$. Let us recall that $\mathbf{G} \in \operatorname{SO}_{\mathrm{o}}(2,1)$ and $\mathbf{U} \in \operatorname{SL}(2, \mathbb{R})$ satisfy Eqs. (63) and (64) with $\mathbf{A} \in \operatorname{so}(2,1)$ and $\mathbf{B} \in \operatorname{sl}(2, \mathbb{R})$.

For the spin transformation $\mathbf{U} \in \mathrm{SL}(2, \mathbb{R})$, parametrizing Eq. (44) as

$$
\mathbf{H}(t)=\mathbf{U}(t) \mathbf{H}(0) \mathbf{U}^{\mathrm{t}}(t),
$$

differentiating Eq. (65) with respect to the parameter, and using Eqs. (64) and (65) again, we obtain

$$
\left[\begin{array}{ll}
\dot{H}_{11} & \dot{H}_{12} \\
\dot{H}_{12} & \dot{H}_{22}
\end{array}\right]=\left[\begin{array}{ll}
B_{11} & B_{12} \\
B_{21} & B_{22}
\end{array}\right]\left[\begin{array}{ll}
H_{11} & H_{12} \\
H_{12} & H_{22}
\end{array}\right]+\left[\begin{array}{ll}
H_{11} & H_{12} \\
H_{12} & H_{22}
\end{array}\right]\left[\begin{array}{ll}
B_{11} & B_{21} \\
B_{12} & B_{22}
\end{array}\right] .
$$

Also parametrizing Eq. (48) as

$$
\left[\begin{array}{l}
H_{11}(t) \\
H_{12}(t) \\
H_{22}(t)
\end{array}\right]=\mathbf{R}(t)\left[\begin{array}{l}
H_{11}(0) \\
H_{12}(0) \\
H_{22}(0)
\end{array}\right]
$$

taking time derivative of Eq. (67), and then using Eq. (67) again, we have

$$
\left[\begin{array}{c}
\dot{H}_{11} \\
\dot{H}_{12} \\
\dot{H}_{22}
\end{array}\right]=\dot{\mathbf{R}} \mathbf{R}^{-1}\left[\begin{array}{c}
H_{11} \\
H_{12} \\
H_{22}
\end{array}\right]
$$

Comparing the above equation with Eq. (66) yields

$$
\dot{\mathbf{R}} \mathbf{R}^{-1}=\left[\begin{array}{ccc}
2 B_{11} & 2 B_{12} & 0 \\
B_{21} & B_{11}+B_{22} & B_{12} \\
0 & 2 B_{12} & 2 B_{22}
\end{array}\right] .
$$

For the proper orthochronous Lorentz transformation $\mathbf{G} \in \mathrm{SO}_{\mathrm{o}}(2,1)$, taking the time derivative of Eq. (53), and using Eqs. (63), (68) and (53) again, we obtain

$$
\mathbf{A}=\mathbf{C}^{-1}\left[\begin{array}{ccc}
2 B_{11} & 2 B_{12} & 0 \\
B_{21} & B_{11}+B_{22} & B_{12} \\
0 & 2 B_{12} & 2 B_{22}
\end{array}\right] \mathbf{C} \text {. }
$$

The inverse relation is 


$$
\left[\begin{array}{ccc}
2 B_{11} & 2 B_{12} & 0 \\
B_{21} & B_{11}+B_{22} & B_{12} \\
0 & 2 B_{12} & 2 B_{22}
\end{array}\right]=\mathbf{C A C}^{-1}
$$

Substituting Eq. (47) for $\mathbf{C}$ and Eq. (50) for $\mathbf{C}^{-1}$ in Eq. (69) yields

$$
\left[\begin{array}{lll}
A_{11} & A_{12} & A_{10} \\
A_{21} & A_{22} & A_{20} \\
A_{01} & A_{02} & A_{00}
\end{array}\right]=\left[\begin{array}{ccc}
0 & B_{12}-B_{21} & B_{11}-B_{22} \\
B_{21}-B_{12} & 0 & B_{12}+B_{21} \\
B_{11}-B_{22} & B_{12}+B_{21} & 0
\end{array}\right]
$$

in which $B_{11}+B_{22}=0$ has been used. Similarly, from Eq. (70) it follows

$$
\left[\begin{array}{ll}
B_{11} & B_{12} \\
B_{21} & B_{22}
\end{array}\right]=\left[\begin{array}{cc}
\frac{1}{4}\left(A_{11}+A_{00}+A_{10}+A_{01}\right) & \frac{1}{2}\left(A_{12}+A_{02}\right) \\
\frac{1}{2}\left(A_{21}+A_{20}\right) & \frac{1}{4}\left(A_{11}+A_{00}-A_{10}-A_{01}\right)
\end{array}\right] .
$$

These formulae relating the matrices $\mathbf{A}$ and $\mathbf{B}$ express explicitly the Lie algebra isomorphism $\operatorname{sl}(2, \mathbb{R}) \cong \operatorname{so}(2,1)$ of the two Lie algebras $\operatorname{sl}(2, \mathbb{R})$ and $\operatorname{so}(2,1)$. Both the Lie algebras have three independent parameters.

\section{Jaumann's $U$ and $G$ are disconjugate}

Substituting Eq. (37) for $\mathbf{A}$ in Eq. (72) gives

$$
\mathbf{B}=\frac{\dot{\gamma}}{2}\left[\begin{array}{cc}
0 & \frac{1}{\gamma_{y}}+f \\
\frac{1}{\gamma_{y}}-f & 0
\end{array}\right] .
$$

Thus we may examine the second order system

$$
\dot{\mathbf{Y}}=\mathbf{B Y}
$$

where $\mathbf{Y}=\left(Y^{1}, Y^{2}\right)^{\mathrm{t}}$, instead of the third order system (24). Eq. (74) can be further recast to the SturmLiouville form

$$
\left(p(\gamma) u^{\prime}(\gamma)\right)^{\prime}+q(\gamma) u(\gamma)=0
$$

either with

$$
u(\gamma)=Y^{1}(\gamma), \quad p(\gamma)=\frac{2 \gamma_{y}}{1+\gamma_{y} f(\gamma)}, \quad q(\gamma)=\frac{1}{2}\left(f(\gamma)-\frac{1}{\gamma_{y}}\right),
$$

or with

$$
u(\gamma)=Y^{2}(\gamma), \quad p(\gamma)=\frac{2 \gamma_{y}}{\gamma_{y} f(\gamma)-1}, \quad q(\gamma)=\frac{1}{2}\left(f(\gamma)+\frac{1}{\gamma_{y}}\right) .
$$

Let $c$ denote a real constant. A function $u(\gamma)$ defined on the interval $I:=[\alpha, \beta), 0 \leqslant \alpha<\beta \leqslant \infty$ or a solution $u(\gamma)$ of Eq. (75) on $I$ is oscillatory (resp. non-oscillatory) if $u(\gamma)$ is real-valued and nontrivial $(\not \equiv 0)$ and $u(\gamma)-c$ has an infinite (resp. a finite) number of zeros on $I$. Specifically, a non-oscillatory solution $u(\gamma)$ of Eq. (75) is said to be disconjugate if $u(\gamma)-c$ has at most one zero. Eq. (75) is said to be oscillatory on $I$ if at least one real-valued nontrivial solution $u(\gamma)$ is such that $u(\gamma)-c$ has an infinite number of zeros on $I$. Conversely, when every real-valued nontrivial solution $u(\gamma)$ is such that $u(\gamma)-c$ has at most a finite number of zeros on $I, u(\gamma)$ is said to be non-oscillatory on $I$. Furthermore, in the latter case, Eq. (75) is said to be disconjugate on $I$ if every real-valued nontrivial solution $u(\gamma)$ is such that $u(\gamma)-c$ has at most one zero on $I$. 
We call a fundamental solution $\mathbf{U}$ satisfying Eq. (64) disconjugate (resp. non-oscillatory, oscillatory) on the interval $I$ if every (resp. every, at least one) component of $\mathbf{U}$ is disconjugate (resp. non-oscillatory, oscillatory) on the interval $I$. Similarly, a fundamental solution G satisfying Eq. (63) is said to be disconjugate (resp. non-oscillatory, oscillatory) on the interval $I$ if every (resp. every, at least one) component of $\mathbf{G}$ is disconjugate (resp. non-oscillatory, oscillatory) on the interval $I$. We call stress $\mathbf{s}$ non-oscillatory (resp. oscillatory) on the interval $I$ if every (resp. at least one) component of $\mathbf{s}$ is non-oscillatory (resp. oscillatory) on the interval $I$, but for stress we will not speak of disconjugacy because the stress responses may depend heavily on the initial stresses; thus the condition of disconjugacy for stress would have been too restricted and tedious to make sense.

Now let us turn our attention to the Jaumann equation and seek the closed-form formulae for $\mathbf{U}$ and the corresponding $\mathbf{G}$ for the plastic phase. Let us substitute $f=1$ into Eq. (73) and solve Eq. (64) for

$$
\mathbf{U}_{\mathbf{J}}(\gamma)=\left[\begin{array}{cc}
\cosh \frac{m \gamma}{2} & \sqrt{\frac{1+\gamma_{y}}{1-\gamma_{y}}} \sinh \frac{m \gamma}{2} \\
\sqrt{\frac{1-\gamma_{y}}{1+\gamma_{y}}} \sinh \frac{m \gamma}{2} & \cosh \frac{m \gamma}{2}
\end{array}\right] .
$$

For such $\mathbf{U}_{\mathbf{J}}(\gamma)$ we check that det $\mathbf{U}_{\mathbf{J}}(\gamma)=1$ and that $\mathbf{U}_{\mathbf{J}}(\gamma) \in \operatorname{SL}(2, \mathbb{R})$. Then substituting Eq. (78) into Eqs. (54)-(62) we obtain

$$
\mathbf{G}_{\mathbf{J}}(\gamma)=\left[\begin{array}{ccc}
\frac{1-\gamma_{y}^{2} \cosh (m \gamma)}{1-\gamma_{y}^{2}} & \frac{\gamma_{y}}{\sqrt{1-\gamma_{y}^{2}}} \sinh (m \gamma) & \frac{1}{1+\gamma_{y}}[1-\sinh (m \gamma)] \\
-\frac{\gamma_{y}}{\sqrt{1-\gamma_{y}^{2}}} \sinh (m \gamma) & \cosh (m \gamma) & \frac{1}{\sqrt{1-\gamma_{y}^{2}}} \sinh (m \gamma) \\
\frac{1}{1+\gamma_{y}}[\sinh (m \gamma)-1] & \frac{1}{\sqrt{1-\gamma_{y}^{2}}} \sinh (m \gamma) & \frac{\cosh (m \gamma)-\gamma_{y}^{2}}{1-\gamma_{y}^{2}}
\end{array}\right] .
$$

We check that the above $\mathbf{G}_{\mathbf{J}}(\gamma)$ satisfies properties (39)-(41); i.e., $\mathbf{G}_{\mathbf{J}} \in \mathrm{SO}_{\mathrm{o}}(2,1)$. In view of formulae (78) and (79), and also of formulae (31) and (21), we obtain immediately the following:

Lemma 1. For the simple shear problem of perfect elastoplasticity using the Jaumann rate, the fundamental solutions $\mathbf{U}_{\mathbf{J}}$ and $\mathbf{G}_{\mathbf{J}}$ are disconjugate for $0 \leqslant \gamma<\infty$ and the stress responses $s^{11}$ and $s^{12}$ are non-oscillatory for $0 \leqslant \gamma<\infty$.

\section{Non-oscillation criterion}

Consider the following two differential equations:

$$
\left(p_{j}(\gamma) u^{\prime}(\gamma)\right)^{\prime}+q_{j}(\gamma) u(\gamma)=0, \quad j=1,2,
$$

where $p_{j}(\gamma)$ and $q_{j}(\gamma)$ are real-valued continuous functions of $\gamma$ on an interval $I=[\alpha, \beta), 0 \leqslant \alpha<\beta \leqslant \infty$. If

$$
p_{1}(\gamma) \geqslant p_{2}(\gamma)>0, \quad q_{1}(\gamma) \leqslant q_{2}(\gamma)
$$

then Eq. (80b) is called a Sturm majorant for Eq. (80a) on $I$, and Eq. (80a) is a Sturm minorant for Eq. (80b) on $I$.

The following lemma is an extension of Sturm's comparison theorem (see Liu and Hong, 2000).

Lemma 2. Let Eq. (80b) be a Sturm majorant for Eq. (80a). If Eq. (80b) is disconjugate on the interval I, then Eq. (80a) is also disconjugate on the interval I.

Now let us consider an objective corotational stress rate $\stackrel{\text { s }}{\mathbf{2}}$ with the general plane spin $\omega$ of Eq. (21) and give a sufficient condition for the stress responses to be non-oscillatory. 
Theorem 1 (Non-oscillation criterion). For the simple shear problem of a perfectly elastoplastic body, suppose that the function $f(\gamma)$ in Eq. (21) is continuous on the interval $\mathbb{R}^{+}:=[0, \infty)$. If $f(\gamma) \leqslant 1$ on the interval $\mathbb{R}^{+}$, then the fundamental solution $\mathbf{U}$ satisfying Eq. (64) with $\mathbf{B}$ given by Eq. (73) is disconjugate on the interval $\mathbb{R}^{+}$, and the fundamental solution $\mathbf{G}$ satisfying Eq. (63) with A given by Eq. (37) is non-oscillatory on the interval $\mathbb{R}^{+}$. Hence the stress responses $s^{11}$ and $s^{12}$ for such $f(\gamma)$ are non-oscillatory on the interval $\mathbb{R}^{+}$.

Proof. Recall that for the Jaumann rate, $f(\gamma)=f_{\mathrm{J}}(\gamma)=1$. If $f(\gamma) \leqslant f_{\mathrm{J}}(\gamma)$, according to Eq. (76) (or Eq. (77)) we have $p(\gamma) \geqslant p_{\mathrm{J}}(\gamma)$ and $q(\gamma) \leqslant q_{\mathrm{J}}(\gamma)$. Then the comparison made between Eq. (76) or (77) with $f(\gamma)=f_{\mathrm{J}}(\gamma)$ and those with $f(\gamma) \leqslant f_{\mathrm{J}}(\gamma)$ shows that the Jaumann equation is a Sturm majorant for the equation with $f(\gamma) \leqslant f_{\mathbf{J}}(\gamma)$. Because, according to Lemma $1, \mathbf{U}_{\mathbf{J}}$ and $\mathbf{G}_{\mathbf{J}}$ are disconjugate, by Lemma 2 the fundamental solution $\mathbf{U}$ of Eq. (74) for $f(\gamma) \leqslant f_{\mathrm{J}}(\gamma)$ is disconjugate and the $\mathbf{G}$ for $f(\gamma) \leqslant f_{\mathrm{J}}(\gamma)$ obtained from Eqs. (56)(62) via the multiplication, addition, and subtraction of the disconjugate functions is non-oscillatory, not necessarily disconjugate, however. The solution of Eq. (24) can be expressed in the following transition formula:

$$
\mathbf{X}(\gamma)=\left[\mathbf{G}(\gamma) \mathbf{G}^{-1}\left(\gamma_{\text {on }}\right)\right] \mathbf{X}\left(\gamma_{\text {on }}\right), \quad \forall \gamma \geqslant \gamma_{\text {on }},
$$

where $\mathbf{X}:=\left(X^{1}, X^{5}, X^{0}\right)^{\mathrm{t}}$ is the augmented stress. We solve Eq. (39) for the inverse

$$
\mathbf{G}^{-1}=\mathbf{g G}^{\mathrm{t}} \mathbf{g}
$$

and partition $\mathbf{G}$ as

$$
\mathbf{G}=\left[\begin{array}{ll}
\mathbf{G}_{s}^{s} & \mathbf{G}_{0}^{s} \\
\mathbf{G}_{s}^{0} & G_{0}^{0}
\end{array}\right]
$$

where $\mathbf{G}_{s}^{s}, \mathbf{G}_{0}^{s}$ and $\mathbf{G}_{s}^{0}$ are of order $2 \times 2,2 \times 1$ and $1 \times 2$, respectively. Thus Eq. (82) can be partitioned as follows:

$$
\left[\begin{array}{l}
\mathbf{X}^{s}(\gamma) \\
X^{0}(\gamma)
\end{array}\right]=\left[\begin{array}{ll}
\mathbf{G}_{s}^{s}(\gamma)\left(\mathbf{G}_{s}^{s}\right)^{\mathrm{t}}\left(\gamma_{\text {on }}\right)-\mathbf{G}_{0}^{s}(\gamma)\left(\mathbf{G}_{0}^{s}\right)^{\mathrm{t}}\left(\gamma_{\text {on }}\right) & \mathbf{G}_{0}^{s}(\gamma) G_{0}^{0}\left(\gamma_{\text {on }}\right)-\mathbf{G}_{s}^{s}(\gamma) \mathbf{G}_{s}^{0}\left(\gamma_{\text {on }}\right) \\
\mathbf{G}_{s}^{0}(\gamma)\left(\mathbf{G}_{s}^{s}\right)^{\mathrm{t}}\left(\gamma_{\text {on }}\right)-G_{0}^{0}(\gamma)\left(\mathbf{G}_{0}^{s}\right)^{\mathrm{t}}\left(\gamma_{\text {on }}\right) & G_{0}^{0}(\gamma) G_{0}^{0}\left(\gamma_{\text {on }}\right)-\mathbf{G}_{s}^{0}(\gamma) \mathbf{G}_{s}^{0}\left(\gamma_{\text {on }}\right)
\end{array}\right]\left[\begin{array}{l}
\mathbf{X}^{s}\left(\gamma_{\text {on }}\right) \\
X^{0}\left(\gamma_{\text {on }}\right)
\end{array}\right]
$$

which is valid for the plastic phase. Substituting the above $\mathbf{X}(\gamma)$ into Eq. (25) we obtain the stresses $s^{11}$ and $s^{12}$. Because $X^{0}>0$ is a monotonic increasing function of $\gamma$ and the components of $\mathbf{G}$ are non-oscillatory on the interval $\left[\gamma_{\text {on }}, \infty\right)$, it follows that $s^{11}$ and $s^{12}$ are non-oscillatory on the interval $\left[\gamma_{\text {on }}, \infty\right)$. On the interval $\left[0, \gamma_{\text {on }}\right)$, the body is in the elastic phase, and the governing equations reduce to hypoelasticity equations, $\dot{\mathbf{s}}-\boldsymbol{\omega} \mathbf{s}+\mathbf{s} \boldsymbol{\omega}=2 G \mathbf{D}$, which render the stress responses non-oscillatory as proved in Liu and Hong (2000). Therefore, the stress responses are non-oscillatory for $\gamma \in[0, \infty)$.

\section{The Jaumann equation is a Sturm majorant}

Now let us compare the five stress rates listed in Section 5. From the expressions for the five $f$ 's in Section 5 (and Fig. 1), we have the following inequalities:

$$
\begin{aligned}
& f_{\mathrm{GN}} \leqslant f_{\mathrm{J}}, \quad \forall \gamma \in \mathbb{R}^{+}, \\
& f_{\mathrm{SC}}<f_{\mathrm{J}}, \quad \forall \gamma \in \mathbb{R}^{+}, \\
& f_{\mathrm{XBM}} \leqslant f_{\mathrm{J}}, \quad \forall \gamma \in \mathbb{R}^{+}, \\
& f_{\mathrm{LMW}} \leqslant f_{\mathrm{J}}, \quad \forall \gamma \geqslant 1 .
\end{aligned}
$$


Then from Eq. (76) or (77) it follows that

$$
\begin{aligned}
& p_{\mathrm{GN}} \geqslant p_{\mathrm{J}}, \quad q_{\mathrm{GN}} \leqslant q_{\mathrm{J}}, \quad \forall \gamma \in \mathbb{R}^{+}, \\
& p_{\mathrm{SC}}>p_{\mathrm{J}}, \quad q_{\mathrm{SC}}<q_{\mathrm{J}}, \quad \forall \gamma \in \mathbb{R}^{+}, \\
& p_{\mathrm{XBM}} \geqslant p_{\mathrm{J}}, \quad q_{\mathrm{XBM}} \leqslant q_{\mathrm{J}}, \quad \forall \gamma \in \mathbb{R}^{+}, \\
& p_{\mathrm{LMW}} \geqslant p_{\mathrm{J}}, \quad q_{\mathrm{LMW}} \leqslant q_{\mathrm{J}}, \quad \forall \gamma \geqslant 1,
\end{aligned}
$$

confirming that the Jaumann equation is a Sturm majorant for the equations of Green-Naghdi, SowerbyChu and Xiao-Bruhns-Meyers on the interval $\mathbb{R}^{+}$, and of Lee-Mallett-Wertheimer in the range $\gamma \geqslant 1$. Then using Lemmas 1 and 2 and Theorem 1 we obtain the following

Theorem 2. For the simple shear problem of a perfectly elastoplastic body, the Jaumann rate renders the stress responses non-oscillatory for $\gamma \in \mathbb{R}^{+}$. Moreover, the Jaumann equation is a Sturm majorant for the equations of Green-Naghdi, Sowerby-Chu and Xiao-Bruhns-Meyers for $\gamma \in \mathbb{R}^{+}$, and of Lee-Mallett-Wertheimer for $1 \leqslant \gamma<\infty$. Therefore, the rates of Green-Naghdi, Sowerby-Chu, and Xiao-Bruhns-Meyers render the stress responses non-oscillatory for $\gamma \in \mathbb{R}^{+}$, and the Lee-Mallett-Wertheimer rate renders the stress responses nonoscillatory for $1 \leqslant \gamma<\infty$.

Fig. 3 displays the stress response curves of the model of perfect elastoplasticity with the five objective corotational stress rates, which as can be seen are located in a narrow strip between Jaumann's and Sowerby-Chu's curves. The strips have a width of about $0.07 \tau_{y}$ for the axial stress $s^{11}$ and about $0.002 \tau_{y}$ for the shear stress $s^{12}$, indicating that the differences of $f$ 's have merely a minor influence on the responses. Indeed, as already mentioned, in Eq. (24) $f$ is much less than $1 / \gamma_{y}$; therefore, the control matrix $\mathbf{A}$ in the plastic phase (and also in the elastic phase) is dominated by $1 / \gamma_{y}$, not by $f$. Fig. 3(b) shows that the LeeMallett-Wertheimer rate renders slightly oscillatory responses in the initial stage. This may be attributed to the fact that $f_{\mathrm{LMW}}>f_{\mathrm{J}}$ for $\gamma<1$. However, its responses from $\gamma=1$ on must not oscillate as guaranteed by Theorem 2 and do not oscillate as shown in Fig. 3(b).

Moss (1984) was the first to point out that for the stress rates of Jaumann and Green-Naghdi "instability" may occur in the shear stress component at the elastic-plastic transition point of the model of perfect elastoplasticity (see Fig. 3(b)). He also noted that the instability was due to the "inaccuracy" of the structure of the mathematical theory. However, as illustrated in Fig. 5, where the responses based on the Green-Naghdi rate with two sets of initial conditions are compared and, in particular, the alleged "instability" disappears in the responses of the model which has been pre-stressed to $s^{11}=200 \mathrm{MPa}$ before simple shearing, it would be clear that oscillation or the so-called instability depends in fact on the initial conditions prescribed, but not at all on the "inaccuracy" of the mathematical theory.

\section{Conclusions}

For the simple shear problem of a perfectly elastoplastic body, we conclude that:

1. The rates of Jaumann, Green-Naghdi, Sowerby-Chu, and Xiao-Bruhns-Meyers render the stress responses non-oscillatory for the shear engineering strain $\gamma \in \mathbb{R}^{+}$, while the Lee-Mallett-Wertheimer rate renders the stress responses non-oscillatory for $1 \leqslant \gamma<\infty$.

2. The Jaumann equation is a Sturm majorant for the equations of Green-Naghdi, Sowerby-Chu, and Xiao-Bruhns-Meyers for $\gamma \in \mathbb{R}^{+}$, and of Lee-Mallett-Wertheimer for $1 \leqslant \gamma<\infty$, and so the non-oscillation of the Jaumann equation implies the non-oscillation of the other equations. 

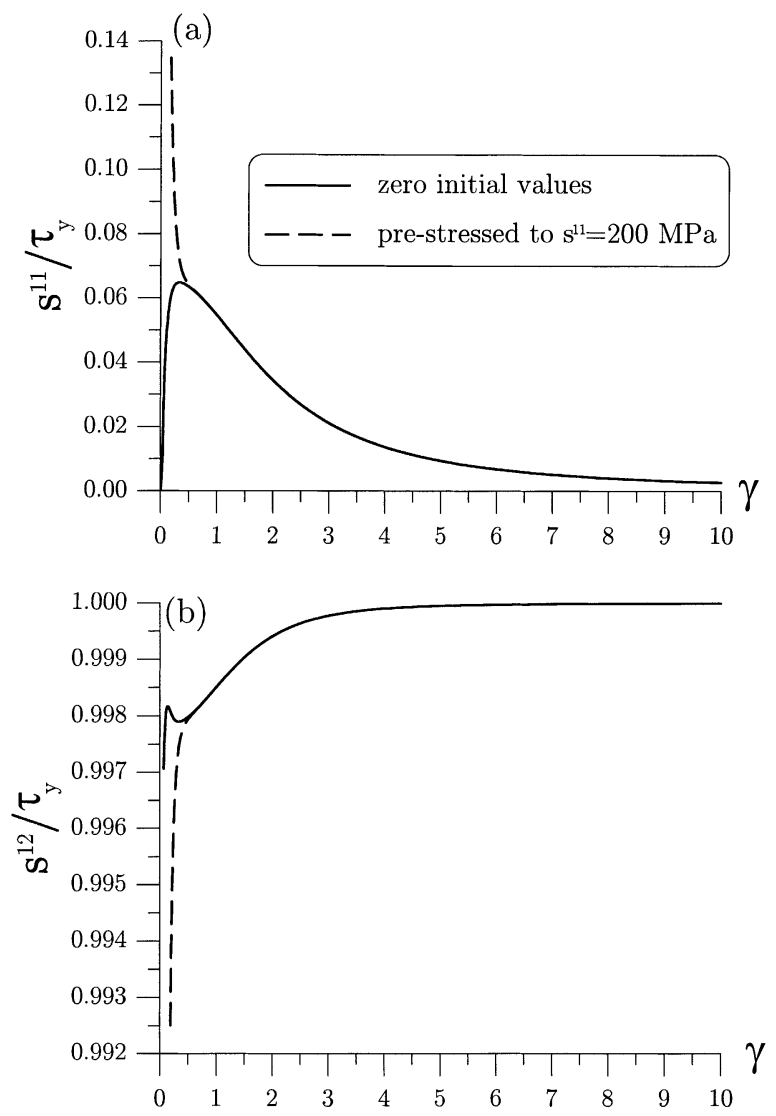

Fig. 5. Influences of initial stresses on the responses (of the model of perfect elastoplasticity using the Green-Naghdi stress rate): zero initial stresses versus pre-stress $s^{11}=200 \mathrm{MPa}$. Notice that the alleged "instability" of the shear stress response in the elastic-plastic transition does not exist for the pre-stress case.

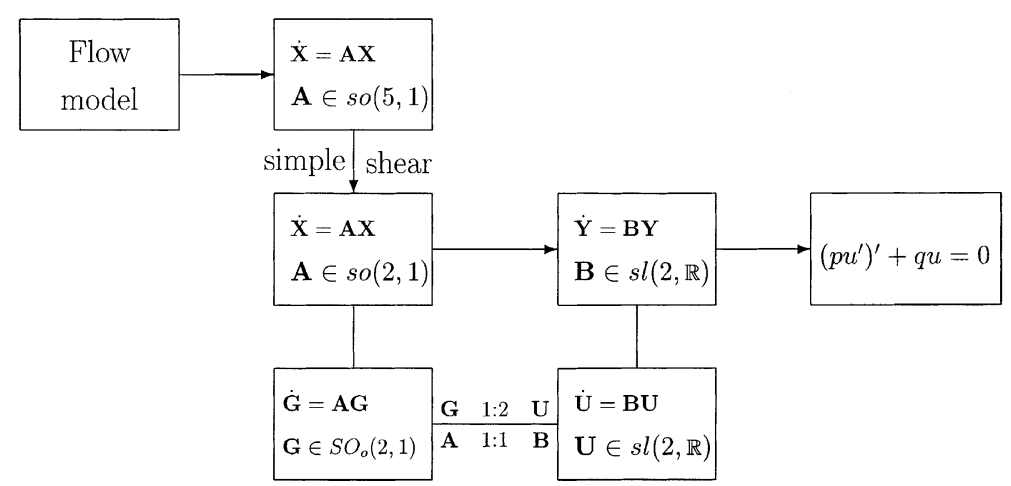

Fig. 6. Flow chart for the methodology of the present paper.

3. For an objective corotational stress rate with the general plane spin the condition $1 \geqslant f(\gamma) \in C\left(\mathbb{R}^{+}\right)$is sufficient to ensure the stress responses to be non-oscillatory on the interval $\mathbb{R}^{+}$. In other words, to obtain 
non-oscillatory stress responses, the plane spin $\omega_{12}$ in the objective corotational stress rate used must not exceed the shear strain rate $\dot{\gamma} / 2$ exerted to the body.

4. For the Jaumann rate the fundamental difference of the mathematical structures of the augmented stress equations between hypoelasticity and elastoplasticity and the physical limit of the shear yield engineering strain $0<\gamma_{y}<1$ are the major factors causing their stress responses qualitatively different.

To reach such conclusions we performed a Sturm-type qualitative analysis of the related equations resulting from a series of exact conversions summarized in Fig. 6. We converted the constitutive equations into a third order linear system, and developed a consistency scheme to calculate the responses. Then, using the Lie algebra isomorphism of $\mathrm{sl}(2, \mathbb{R})$ onto $\mathrm{so}(2,1)$ we found a second order linear system representation, which in turn was converted into a Sturm-Liouville equation. This series of conversions involves no approximations at all and is essential for the success of the qualitative analysis.

The main tools of this paper were the exact linearization of the flow model, the reduction of the third order linear system to the second order linear system, and the extension of the Sturm comparison theorem at the first time to qualitatively assess the effects of different corotational stress rates on the responses of a perfectly elastoplastic body. This methodology may be extended to investigate the simple shear problem of other more complicated models.

\section{References}

Atluri, S.N., 1984. On constitutive relations at finite strain: Hypoelasticity and elastoplasticity with isotropic or kinematic hardening. Comp. Meth. Appl. Mech. Engng. 43, 137-171.

Bruhns, O.T., Xiao, H., Meyers, A., 1999. Self-consistent Eulerian rate type elastoplasticity models based upon the logarithmic stress rate. Int. J. Plasticity $15,479-520$.

Dienes, J.K., 1979. On the analysis of rotation and stress rate in deforming bodies. Acta Mech. 32, $217-232$.

Green, A.E., Naghdi, P.M., 1965. A general theory of an elastic-plastic continuum. Arch. Rat. Mech. Anal. 18, $251-281$.

Hirth, J.P., Lothe, J., 1982. Theory of Dislocations. Second edition. Wiley, New York.

Hong, H.-K., Liu, C.-S., 1999. Lorentz group $\mathrm{SO}_{\mathrm{o}}(5,1)$ for perfect elastoplasticity with large deformation and a consistency scheme. Int. J. Non-Linear Mech. 34, 1113-1130.

Hong, H.-K., Liu, C.-S., 2000. Internal symmetry in the constitutive model of perfect elastoplasticity. Int. J. Non-Linear Mech. 35, $447-466$.

Jaumann, G., 1911. Geschlossenes system physikalischer und chemischer differentialgesetze. Sitzber. Akad. Wiss. Wien 120, 385-530.

Johnson, G.C., Bammann, D.J., 1984. A discussion of stress rates in finite deformation problems. Int. J. Solids Struct. $20,725-737$.

Lee, E.H., Mallett, R.L., Wertheimer, T.B., 1983. Stress analysis for anisotropic hardening in finite-deformation plasticity. J. Appl. Mech. ASME 50, 554-560.

Lehmann, Th., Guo, Z.-H., Liang, H.Y., 1991. The conjugacy between Cauchy stress and logarithm of the left stretch tensor. Eur. J. Mech. A/Solids 10, 395-404.

Liu, C.-S., Hong, H.-K., 2000. Non-oscillation criteria for hypoelastic models under simple shear deformation. J. Elasticity, to appear.

Metzger, D.R., Dubey, R.N., 1987. Corotational rates in constitutive modeling of elastic-plastic deformation. Int. J. Plasticity 3, 341368.

Moss, W.C., 1984. On instabilities in large deformation simple shear loading. Comp. Meth. Appl. Mech. Engng. 46, 329-338.

Nagteggal, J.C., de Jong, J.E., 1982. Some aspects of non-isotropic work-hardening in finite strain plasticity. In: Lee, E.H., Mallett, R.L. (Eds.), Proceedings of the Workshop on Plasticity of Metals at Finite Strain: Theory, Experiment and Computation, Stanford University, pp. 65-102.

Reinhardt, W.D., Dubey, R.N., 1996. Coordinate-independent representation of spins in continuum mechanics. J. Elasticity 42, 133144.

Sowerby, R., Chu, E., 1984. Rotations, stress rate and strain measures in homogeneous deformation processes. Int. J. Solids Struct. 20, $1037-1048$.

Szabó, L., Balla, M., 1989. Comparison of some stress rates. Int. J. Solids Struct. 25, 279-297.

Xiao, H., Bruhns, O.T., Meyers, A., 1997a. Hypoelasticity model based upon the logarithmic stress rate. J. Elasticity 47, 51-68.

Xiao, H., Bruhns, O.T., Meyers, A., 1997b. Logarithmic strain, logarithmic spin and logarithmic rate. Acta Mech. 124, 89-105.

Yang, W., Cheng, L., Hwang, K.-C., 1992. Objective corotational rates and shear oscillation. Int. J. Plasticity 8, $643-656$. 